\title{
The Early Spectrophotometric Evolution of V1186 Scorpii (Nova Scorpii 2004 \#1)
}

\author{
G. J. Schwarz ${ }^{1}$, C. E. Woodward ${ }^{2}$, M. F. Bode ${ }^{3}$, A. Evans ${ }^{4}$, S. P. Eyres ${ }^{5}$, T. R. Geballe ${ }^{6}$ \\ R. D. Gehrz ${ }^{2}$, M. A. Greenhouse ${ }^{7}$, L. A. Helton ${ }^{2}$, W. Liller ${ }^{8}$, J. E. Lyke ${ }^{9}$, D. K. Lynch ${ }^{10,18}$, \\ T. J. O'Brien ${ }^{11}$, R. J. Rudy ${ }^{10,18}$, R. W. Russell ${ }^{10,18}$, S. N. Shore ${ }^{12}$, S. G. Starrfield ${ }^{13}$, T. \\ Temim $^{2}$, J. W. $\operatorname{Truran}^{14}$, C. C. Venturini ${ }^{10}$, R. M. Wagner ${ }^{15}$ R. E. Williams ${ }^{16}$, R. \\ Zamanov ${ }^{3,17}$
}

\footnotetext{
${ }^{1}$ West Chester University, Department of Geology and Astronomy, 750 S. Church St., West Chester, PA 19383,gschwarz@pha.jhu.edu

${ }^{2}$ Department of Astronomy, School of Physics and Astronomy, 116 Church Street, S. E., University of Minnesota, Minneapolis, MN 55455

${ }^{3}$ Astrophysics Research Institute, Liverpool John Moores University, Twelve Quays House, Birkenhead CH41 1LD, UK

${ }^{4}$ Astrophysics Group, Keele University, Keele, Staffordshire ST5, 5BG, UK

${ }^{5}$ Department of Physics, Astronomy and Mathematics, University of Central Lancashire, Preston PR1 $2 \mathrm{HE}, \mathrm{UK}$

${ }^{6}$ Gemini Observatory, 670 North A'ohoku Place, Hilo, HI 96720

${ }^{7}$ NASA Goddard Space Flight Center, Code 665, Greenbelt, MD 20771

${ }^{8}$ Institute for Nova Studies, Casilla 5022, Viña del Mar, Chile

${ }^{9}$ W. M. Keck Observatory, 65-1120 Mamalahoa Hwy., Kamuela, HI 96743

${ }^{10}$ The Aerospace Corporation, Mail Stop 2-266, P.O. Box 92957, Los Angeles, CA 90009-2957

${ }^{11}$ Jodrell Bank Observatory, University of Manchester, Macclesfield Cheshire SK11 9DL, UK

${ }^{12}$ Dipartimento di Fisica "Enrico Fermi," Universita' di Pisa, largo Pontecorvo 3, Pisa 56127 Italy; INFN - Sezione di Pisa

${ }^{13}$ School of Earth and Space Exploration, Arizona State University, P.O. Box 871404, Tempe, AZ 85287

${ }^{14}$ Department of Astronomy and Astrophysics, University of Chicago, 5640 S. Ellis Avenue, Chicago, IL 60637 and Argonne National Laboratory, 9700 South Cass Road, Argonne, IL 60439

${ }^{15}$ Large Binocular Telescope Observatory, University of Arizona, 933 North Cherry Avenue, Tucson, AZ 85721
}

${ }^{16}$ Space Telescope Science Institute, 3700 San Martin Drive, Baltimore, MD 21218

${ }^{17}$ Bulgarian National Astronomy Institute

${ }^{18}$ Visiting Astronomer at the Infrared Telescope Facility, which is operated by the University of Hawaii under Cooperative Agreement NCC 5-538 with NASA Office of Space Science, Planetary Astronomy Program 


\begin{abstract}
We report optical photometry and optical through mid-infrared spectroscopy of the classical nova V1186 Sco. This slowly developing nova had an complex light curve with multiple secondary peaks similar to those seen in PW Vul. The time to decline 2 magnitudes, $\mathrm{t}_{2}$, was 20 days but the erratic nature of the light curve makes determination of intrinsic properties based on the decline time (e.g., luminosity) problematic, and the often cited MMRD relationship of della Valle \& Livio (1995) fails to yield a plausible distance. Spectra covering 0.35 to $35 \mu \mathrm{m}$ were obtained in two separate epochs during the first year of outburst. The first set of spectra, taken about 2 months after visible maximum, are typical of a CO-type nova with narrow line emission from H I, Fe II, O I and He I. Later data, obtained between 260 and 380 days after maximum, reveal an emerging nebular spectrum. Spitzer spectra show weakening hydrogen recombination emission with the emergence of [Ne II] $(12.81 \mu \mathrm{m})$ as the strongest line. Strong emission from [Ne III] $(15.56 \mu \mathrm{m})$ is also detected. Photoionization models with low effective temperature sources and only marginal neon enhancement ( $\mathrm{Ne} \sim$ $1.3 \mathrm{Ne}_{\odot}$ ) are consistent with these IR fine-structure neon lines indicating that V1186 Sco did not occur on a ONeMg white dwarf. In contrast, the slow and erratic light curve evolution, spectral development, and photoionization analysis of the ejecta imply the outburst occurred on a low mass CO white dwarf. We note that this is the first time strong [Ne II] lines have been detected so early in the outburst of a CO nova and suggests that the presence of mid-infrared neon lines is not directly indicative of a $\mathrm{ONeMg}$ nova event.
\end{abstract}

Subject headings: stars: individual (V1186 Sco) — stars: novae.

\title{
1. INTRODUCTION
}

Classical nova outbursts occur in binaries consisting of a white dwarf (WD) and a low mass star that has filled its Roche lobe. Matter is accreted onto the WD with the deeper layers becoming degenerate. The temperature increases, first through compression and then rapidly when thermonuclear reactions begin, eventually leading to a thermonuclear runaway. Energy deposited in the accreted mass is sufficient to eject a fraction of the material. The amount of mass accreted and subsequently ejected, along with the energetics of the outburst are related to the mass and composition of the underlying WD. Outbursts on Carbon-Oxygen (CO) WDs tend to eject more mass under less energetic conditions than novae on OxygenNeon-Magnesium (ONeMg) WDs (Schwarz et al. 1997, 2001, 2007). 
V1186 Sco was discovered on 03.1 July 2004 UT by Pojmanski (2004) at V = 11.98. The earliest optical spectrum of V1186 Sco was obtained on 06.51 July 2004 UT, or about 3 days before visual maximum (Fujii 2004). This spectrum displayed strong Balmer emission lines with FWHM of order $500 \mathrm{~km} \mathrm{~s}^{-1}$ and possible P-Cygni profiles, bearing a strong resemblance to the pre-maximum spectra of the slow nova V723 Cas (Evans et al. 2003; Munari et al. 1996). Spitzer Space Telescope Target of Opportunity (ToO) observations were immediately activated and V1186 Sco was observed during two observational epochs in the first year of outburst. The Spitzer data were complemented with contemporaneous optical and near-IR (NIR) spectra obtained as part of a coordinated multi-wavelength nova observing initiative. These data provided wavelength coverage from 0.35 to $35 \mu \mathrm{m}$ with a variety of spectral resolutions. In addition, the early photometric light curve is well sampled showing a leisurely rise and subsequent decline (see $§ 2.1$ ). The light curve behavior, the low expansion velocities, and the similarity of the early spectra of V1186 Sco (Fujii 2004, and \$2.2) to those of V723 Cas (Evans et al. 2003) and PW Vul (Gehrz et al. 1988; Evans et al. 1990; Williams et al. 1996) implies that the outburst occurred on a low mass CO-type WD. The behavior of V1186 Sco contrasts markedly with that of the other nova discovered in the constellation Scorpius in 2004, the fast ONeMg nova V1187 Sco (Lynch et al. 2006). In particular, the development of infrared coronal lines detected with Spitzer was significantly more rapid in V1187 Sco (cf., §4).

We present the photometric and spectroscopic data in $\S 2$. Section 3 discusses the estimate of the reddening and distance to V1186 Sco. Section 4 describes the line evolution, particularly the significant detection of $[\mathrm{Ne}$ II] and [Ne III] in the Spitzer spectra. A photoionization model of the later multiwavelength spectra is also presented in $\S 4$. Section 5 presents a discussion of our primary results followed by our conclusions in $\S 6$.

\section{OBSERVATIONS}

\subsection{Photometry}

The early light curve of V1186 Sco is shown in Fig. 1a, and the first year light curve is presented in Fig. 1 b. The $\mathrm{V}$ band data are from the IAU circulars, VSNET, the AAVSO (Waagen 2005) and the Liverpool Robotic Telescope (Steele et al. 2004)1. Photometric data

\footnotetext{
${ }^{1}$ The 2-m fully robotic Liverpool Telescope is operated on the island of La Palma by Liverpool John Moores University in the Spanish Observatorio del Roque de los Muchachos of the Instituto de Astrofisica de Canarias.
} 
were obtained using the $2048 \times 2048$ CCD camera "RATCam"2 . Photometric data were reduced using the GAIA image analysis software package and are provided in Table 1 .

The V1186 Sco light curve exhibits many interesting features. The first notable feature was a sluggish rise to visual maximum. After discovery, V1186 Sco required 6 days to reach its maximum at $\mathrm{V}=9.6$. Very few novae have exhibited such long pre-maximum behavior which is generally only found in very slow novae. Another intriguing feature of the V1186 Sco light curve is the strength of the second maximum that occurred about 9 days later after the first. This second maximum was extremely bright, only $\sim 0.2$ mag fainter than the primary maximum. If not for the $\sim 1$ mag decline twelve days after discovery, the early light curve evolution might be better described as a plateau, similar to that observed in V723 Cas. While secondary maxima are not unusual in slower nova they do not usually approach that of the primary maximum (see Payne-Gaposchkin 1957, for numerous examples of nova light curves). For example, PW Vul displayed multiple secondary peaks in its light curve but they were all more than a magnitude fainter than at maximum.

After the second peak in the V1186 Sco light curve, the V magnitude showed a rapid decline passing its $t_{2}$ point (the time to decline 2 magnitudes from maximum) in 20 days. Subsequently, the V1186 Sco light curve had two smaller peaks during its decline. These later peaks make it difficult to establish the $t_{3}$ time (the time to decline 3 magnitudes from maximum) since the brightness fluctuated around $\mathrm{V}=12.6$. V1186 Sco first reached $\mathrm{V}=12.6$ after 45 days but then brightened and subsequently did not become fainter than $\mathrm{V}=12.6$ until 70 days after maximum. Which decline value is representative of this nova? The choice has significant consequences on speed-class classification (Warner 1995), WD progenitor identification, and any physical parameters based on the derived decline times. After 100 days the $\mathrm{V}$ band light curve stabilized at about 13.5 magnitudes as the fading continuum was offset by emerging line emission, particularly from [O III] (5007^). According to Duerbeck's study of about 100 galactic novae (Duerbeck 1981), the light curve of V1186 Sco is classified as type $\mathrm{Bb}$ ("decline with major fluctuations, e.g., double or multiple maxima"). The prototypes of this classification are DN Gem and NQ Vul (see Fig. 3 in Duerbeck 1981). There was no evidence for a major visual extinction event characteristic of dust formation in the visible light curve of V1186 Sco during the first $\approx 300$ days. Dust is not frequently found in novae with short $t_{2}$ timescales, V1974 Cyg or V838 Her (Woodward et al. 1997, 1992) being recent examples.

${ }^{2}$ http://telescope.livjm.ac.uk 


\subsection{SPECTROSCOPY}

Multiwavelength spectroscopy, centered around the Spitzer observations, was obtained during two different epochs. The first, called E1, occurred $~ 60$ days after visual maximum between 22 August 2004 and 23 September 2004. This was the earliest that Spitzer data could be obtained after the ToO trigger was requested. The next set of Spitzer observations could not be scheduled and executed until the following year due to object visibility window constraints. This second epoch, called E2, was taken during the transition to the nebular phase, 21 May 2005 - 16 July 2005, or over 261 days after maximum. Table 2 provides the relevant observational information for all the spectral observations while Tables 3 and 4 give the measured line fluxes, uncorrected for reddening, obtained during the E1 (2004; Spitzer only) and E2 (2005; all data) epochs, respectively.

\subsubsection{Spitzer mid-IR}

V1186 Sco was observed by the Spitzer Space Telescope (Gehrz et al. 2007a; Werner et al. 2004) four times during the two observational epochs using the Infrared Spectrograph (Houck et al. 2004, (IRS)). The observations were part of the Cycle 1 classical nova ToO initiative, program identification number (PID) 2333 (PI C. E. Woodward).

Basic Calibrated Data (BCD) products were calibrated and processed with the Spitzer Science Center (SSC) data pipeline. The IRS observations were conducted using the short wavelength $(5.2-14.5 \mu \mathrm{m})$ low resolution module $(\mathrm{SL})$, the short wavelength $(9.9-19.5 \mu \mathrm{m})$ high resolution module $(\mathrm{SH})$, and the long wavelength $(18.7-37.2 \mu \mathrm{m})$ high resolution module (LH). The resolving power for $\mathrm{SL}$ is $R=\lambda / \Delta \lambda \sim 60-128$, while $\mathrm{R} \sim 600$ for $\mathrm{SH}$ and LH. Emission lines observed with the latter modules are marginally resolved.

All Spitzer observations utilized visual PCRS peak-ups on nearby isolated stars to ensure proper placement of the target in the narrow IRS slits. The spectroscopy of V1186 Sco during E1 (AORs 10273536, 10273792, 10274048) consisted of 2 cycles of 6 second ramps in SL (12 seconds on-source), and 6 cycles of 6 second ramps in SH and LH (36 seconds on-source each), while the E2 observations (AORs 10274304, 10275328) employed 2 cycles of 30 second ramps (60 seconds on-source) for SH, 3 cycles of 60 second ramps for LH (180 seconds onsource), and 3 cycles of 6 second ramps (18 seconds on-source) for SL. IRS basic calibrated data products (BCDs) were processed with version 12.0.2 of the IRS pipeline. Descriptions of the IRS instrument and its operation are available in Houck et al. (2004) and Gehrz et al. (2007a). Details of the calibration and raw data processing are specified in the IRS Pipeline 
Description Document, v1.0 3. E1 epoch observations were obtained with a short synoptic cadence (1 - 2 day), and no marked evolution in the spectral behavior of V1186 Sco was noted within the uncertainty of the absolute IRS calibration errors. Thus, the three separate spectra were averaged together to produce a single composite spectrum for analysis.

Bad pixels were interpolated in individual BCDs using bad pixel masks provided by the SSC. Multiple data collection events were obtained at two different positions on the slit using Spitzer's nod functionality. Sky subtraction was only possible for the SL observations, as no dedicated sky observations were performed for the SH and the LH mode observations. Sky subtraction was performed by differencing the two dimensional SL BCDs to remove the background flux contribution. Spectra were then extracted from the background corrected SL data and the SH and LH BCDs with SPICE (version 1.3-beta1) using the default point source extraction widths. The extracted spectra were then combined using a weighted linear mean into a single output data file. At the time of reduction, the errors generated by the SSC pipeline were not reliable and therefore errors were estimated from the standard deviation of the flux at each wavelength bin. The spectral lines detected in the Spitzer data were fit using a non-linear least squares Gaussian routine (the Marquardt method, Bevington \& Robinson 1992) that returns the line center, line amplitude, integrated line flux, continuum amplitude and the slope of the continuum.

\subsubsection{Ground-based Near-IR}

Three telescopes were used to obtain the NIR spectral data. The first observation was obtained at the Keck II telescope using NIRSPEC (McLean et al. 1998). All of the observations were in low-resolution mode with the $42^{\prime \prime}$ by $0.38^{\prime \prime}$ slit. The seeing during the observation was about $0.8^{\prime \prime}$. Multiple spectra using different sorting filters were taken by nodding V1186 Sco and standards (HD 162220 and HD 163633) along the slit at 15" intervals while guiding. The REDSPEC software was used to reduce the nova and standards using standard IR techniques (Joyce 1992). The N-1 filter spectrum was flux calibrated using the standard's VJHK magnitudes normalized to the $1 \mu \mathrm{m}$ point of a $\mathrm{T}_{\text {eff }}=9480 \mathrm{~K}$ blackbody. The other filters were calibrated using the 2MASS magnitudes of the standards. The resulting fluxes are only approximate because of slit losses.

Additional medium-resolution NIR spectroscopy was obtained from the United King-

\footnotetext{
${ }^{3}$ http://ssc.spitzer.caltech.edu/irs/dh/PDD.pdf
} 
dom Infrared Telescope (UKIRT) ${ }^{4}$ using the facility spectrographs CGS4 (Mountain et al. 1990) and UIST (Ramsay Howat et al. 2004) to cover 0.8-1.35 $\mu \mathrm{m}$ and 1.4-5.1 $\mu \mathrm{m}$ bands, respectively, at resolving powers of 500-2000. The spectra were obtained through UIST's $0.24^{\prime \prime}$ slit and CGS4's $0.6^{\prime \prime}$ slit in the conventional stare/nod-along-slit mode. The data were reduced using standard Figaro procedures, utilizing contemporaneous arc lamp spectra for wavelength calibration and spectra of nearby early type bright stars for flux calibration and removal of telluric absorption lines.

The final NIR data set was acquired from the Infrared Telescope Facility (IRTF) using SpeX (Ravner et al. 2003). The data were obtained through $0.8^{\prime \prime}$ by $15^{\prime \prime}$ slits and a $10^{\prime \prime} \mathrm{N}-\mathrm{S}$ nod for background cancellation. Due to the proximity of calibrators (HD 161822 and HD 157486), chopping and extinction corrections were not necessary. SpeXTools (Cushing et al. 2004) was used to reduce the data. Calibrator V band magnitudes and spectral types from the Bright Star Catalog (Hoffleit \& Jaschek 1982) and matched Kurucz (1991, 1994) spectral energy distribution (SED) models were used to create the flux models. The absolute flux scale was set by normalizing to the calibrators' $\mathrm{K}$ band magnitudes. These were calculated from the known $\mathrm{V}$ band magnitudes and (V-K) colors of stars of similar spectral type (Koornneef 1983) as the calibrators.

\subsubsection{Optical}

The first optical spectrum was obtained at the Multiple Mirror Telescope with the Blue Channel Spectrograph (Schmidt et al. 1989). The slit was in long-slit mode (1" by $\left.180^{\prime \prime}\right)$ with a 300 line $/ \mathrm{mm}$ grating centered at $6000 \AA$ giving a resolving power of $\sim 900$. The second optical spectrum was taken at Steward Observatory's Bok Telescope with the B\&C spectrograph. Observations were obtained using a $1.5^{\prime \prime}$ by $240^{\prime \prime}$ slit and the 400 line $/ \mathrm{mm}$ grating with a central wavelength of $5038 \AA$. The standard stars used for the calibration were Feige 34 and HZ 44. All optical data were reduced using standard IRAF packages 5 and spectral extraction techniques.

\footnotetext{
${ }^{4}$ The United Kingdom Infrared Telescope is operated by the Joint Astronomy Centre of behalf of the United Kingdom Particle Physics and Astronomy Research Council.

${ }^{5}$ IRAF is distributed by the National Optical Astronomy Observatories, which are operated by the Association of Universities for Research in Astronomy, Inc., under cooperative agreement with the National Science Foundation.
} 


\section{REDDENING AND DISTANCE}

An accurate determination of the reddening toward a nova is of critical importance for obtaining corrected line ratios and determining other basic parameters such as the distance. Optical spectra obtained by M. Fujii near visual maximum clearly showed Na I D absorption lines indicative of moderate reddening (Fujii 2004). The Na I D absorption line is present in our earliest optical spectrum but because of the complexity of the blended lines a determination of the reddening is not possible. Thus, other indirect methods must be used to determine the reddening toward V1186 Sco.

van den Bergh \& Younger (1987) suggest two ways to estimate the reddening of a nova using the early light curve decline and colors. They report that at maximum and at $t_{2}$ the average intrinsic (B-V) colors for novae are $0.23 \pm 0.06$ and $-0.02 \pm 0.04$, respectively. Using the observed (B-V) colors for V1186 Sco (see the lower portion of Fig. 1 $\mathrm{b}$ ) the E(B-V) values are consistent at $0.45 \pm 0.1$ and $0.47 \pm 0.16$, respectively. However, the value of the line of sight extinction toward the nova inferred from the reddening map of Schlegel et al. (1998) yield a larger value of $\mathrm{E}(\mathrm{B}-\mathrm{V})=0.96$. Unfortunately, both methods have serious limitations. As described in $\S 2.1$, the light curve of V1186 Sco does not exhibit the smooth decline of a typical nova. Instead the light curve has numerous secondary peaks which make a determination of the true decline times problematic while the Schlegel et al. (1998) extinction map is not as reliable at the location of V1186 Sco, e.g. when $b \leq|5|^{\circ}$.

Alternatively one can infer the reddening by comparing the observed emission line ratios to those predicted under optically thin conditions. For hydrogen lines we use the predicted hydrogen line ratios from Hummer \& Storey (1987) at $\mathrm{T}_{e}=10^{4} \mathrm{~K}$ and $\mathrm{N}_{e}=10^{8} \mathrm{~cm}^{-3}$. This is a typical temperature for a nebular gas and the density is consistent with an ejected mass $\sim 10^{-4} \mathrm{M}_{\odot}$ assuming ejecta traveling in a spherical shell with inner and outer velocities of 500 and $1000 \mathrm{~km} \mathrm{~s}^{-1}$. We use the $\operatorname{Pa} \gamma / \mathrm{Br} \gamma$ ratio in the NIR, as the Balmer decrement may not yet be radiative. The observed $\mathrm{Pa} \gamma / \mathrm{Br} \gamma$ was 2.6, corresponding to $\mathrm{E}(\mathrm{B}-\mathrm{V})$ value of 0.43. Another line ratio method uses the ratios of the NIR O I 0.8448 and $1.128 \mu \mathrm{m}$ lines, with a correction from the $1.316 \mu \mathrm{m}$ line, to determine reddening (Rudy et al. 1991). This method produces an $\mathrm{E}(\mathrm{B}-\mathrm{V})=0.45$ using the last NIR data set. With the exception of the Schlegel et al. (1998) extinction map, all the reddening methods converge toward $\mathrm{E}(\mathrm{B}-\mathrm{V})=$ $0.45 \pm 0.1$ which we adopt as the reddening for V1186 Sco.

Most distance estimates for novae depend on the relationship between the maximum magnitude versus the rate of decay of the light curve (MMRD). Using the MMRD of della Valle \& Livio (1995) and $\mathrm{t}_{2}=20$ days provides an $\mathrm{M}_{V}^{\max }=-8$. With the adopted reddening the derived distance is $17.3 \mathrm{kpc}$ or well beyond the Galaxy. It is highly unlikely that V1186 Sco is this distant. Another approach is to assume the same absolute magnitude 
of a nova at a known distance and with similar properties. PW Vul is a good candidate with a comparable light curve and emission line evolution. An expansion parallax measurement for PW Vul (Downes \& Duerbeck 2000) gives $\mathrm{M}_{V}^{\max }=-6.7$. Adopting this absolute magnitude places the V1186 Sco distance at $9.5_{-1.2}^{+1.4} \mathrm{kpc}$. With V1186 Sco's position toward the Galactic center, even this distance puts V1186 Sco on the other side of the Galaxy and is incongruous with the moderate reddening derived above. As we will show in 44.1 , photoionization modeling requires a significantly lower $\mathrm{M}_{V}$, corresponding to a closer distance of $5.5 \mathrm{kpc}$. Given the erratic nature of the light curve and its location near the Galactic center, we adopt this lower distance as the most realistic distance to V1186 Sco.

\section{LINE EVOLUTION}

The line evolution from E1 (2004) to E2 (2005) in the optical, NIR, and far-IR is shown in Figs. 2 through 5. The E1 data are similar in structure to other CO type novae at the $t_{3}$ time. In the optical V1186 Sco was transitioning from the optically thick to an optically thin phase. The Fe II emission typical of early CO nova had faded and the only forbidden lines seen were $[\mathrm{N}$ II] and $[\mathrm{O} \mathrm{I}]$. In the NIR, the spectra were dominated by hydrogen recombination and emission from neutral oxygen and helium. The E1 Spitzer spectra showed strong hydrogen recombination lines and surprisingly, a weak [Ne II] $(12.81 \mu \mathrm{m})$ line. The emergence of this line is typically taken as a sign that the outburst occurred on the surface of an ONeMg WD (Gehrz et al. 1998) although Greenhouse et al. (1988) and Gehrz et al. (1988) and others have argued that strong continuum emission from condensing dust in the ejecta may mask the presence of [Ne II] lines, especially if observations are obtained at low spectral resolution. Previous [Ne II] detections discussed in the literature, V1974 Cyg (Havward et al. 1992), V1187 Sco (Lynch et al. 2006), QU Vul (Gehrz et al. 2007b; Greenhouse et al. 1988; Gehrz, Grasdalen, \& Hackwell 1985), and CP Cru (Lyke et al. 2003), occurred on ONeMg nova with high ejection velocities $\left(\geq 1000 \mathrm{~km} \mathrm{~s}^{-1}\right)$. However, it is unlikely that the progenitor is a ONeMg WD in the V1186 Sco system as the speed class (slow) and expansion velocities from the line widths are more similar to that observed in CO WD outbursts.

During the next epoch all the spectra are different but the hydrogen lines are still prominent. Almost a year after outburst the nova was just entering the nebular phase with the emergence of [O III] in the optical. The [N II] and [O I] lines observed earlier were still present but He I lines also were present. The overall ionization of the ejecta at this point was still low with no typical high ionization lines (e.g., [Fe VII] 6087) present in the optical spectrum. In the E2 NIR spectrum the emission lines had narrowed due to the expansion of the ejecta and neutral oxygen was still present even one year after outburst. The strongest 
line was the He I triplet at $1.08 \mu \mathrm{m}$ which had increased in intensity by more than a factor of 30. A similar increase was observed but earlier, between 130 and 230 days after maximum in PW Vul (Williams et al. 1996). That the line evolution of V1186 Sco was slower than observed in PW Vul, strengthens our earlier contention that V1186 Sco's $\mathrm{M}_{V}$ value must also be fainter than PW Vul's.

By E2, the [Ne II] emission observed earlier was now prominent in the Spitzer spectra along with [Ne III] $(15.56 \mu \mathrm{m})$. The poor SN of our E2 optical spectrum below $0.4 \mu \mathrm{m}$ makes it impossible to determine if there was any corresponding strong [Ne III]. The 18 to $34 \mu \mathrm{m}$ spectrum was very noisy but showed clear detections of H I (9-8, 12-10, 13-10, and 15-11). Other than the hydrogen and neon lines, no other lines were detected in the Spitzer spectra.

To emphasize the slow evolution in V1186 Sco, Fig. 6]compares the Spitzer observations of V1186 Sco with the faster ONeMg nova V1187 Sco (Lynch et al.|2006). V1187 Sco reached visual maximum on 03 August 2003 UT, or approximately one month after V1186 Sco. With $\mathrm{t}_{2} \simeq 9$ days and $\mathrm{t}_{3}=15$ days, its decay rate was significantly faster than V1186 Sco. Spitzer spectra of both novae in Scorpius were obtained within a few days of each other. The first epoch Spitzer spectra of V1187 Sco (see Fig. 6a) already displayed [Ne II] in addition to higher ionization lines such as [Ne VI] $(7.65 \mu \mathrm{m})$ and $[\mathrm{Ne} \mathrm{V}](14.3 \mu \mathrm{m})$. In the second epoch observations of V1187 Sco (Fig. 6b) the hydrogen emission is almost gone and the higher ionization state lines continued to increase in strength. In addition to the prominent neon lines, V1187 Sco had strong magnesium and argon lines typical of a fast ONeMg nova.

\subsection{PHOTOIONIZATION MODELING}

The available data set poses challenges for photoionization modeling. Due to its lethargic evolution, V1186 Sco was only entering the nebular phase in the E2 (2005) epoch. The majority of the dominant lines are hydrogen recombination lines with relatively few forbidden lines available for analysis. Another dilemma is that there currently are no additional published data sets obtained at later epochs (post-2005) to verify any model solution of the E2 observations. Finally, the E2 data set was obtained over a 100 day span. However, despite these issues it is possible to determine an abundance solution by 1) assuming that the conditions in the ejecta evolved slowly enough that a single model is appropriate for all the spectra obtained during the E2 epoch and 2) fitting the emission lines from each spectral region relative to itself (e.g., optical to $\mathrm{H} \beta$, NIR to $\mathrm{Pa} \beta$, and the Spitzer data to Hu $\alpha$ ) to account for uncertainties in the absolute flux calibrations and the different times the data were obtained. 
We used the Cloudy 2006b photoionization code (Ferland et al. 1998) and assumed a spherical shell ejection with inner and outer radii set by material moving at 500 and 1000 $\mathrm{km} \mathrm{s}^{-1}$ over 260 days. The density profiles assumes $\mathrm{a}^{-3}$ profile to provide a constant mass per unit volume throughout the model shell. The model parameters that were allowed to vary were the effective temperature and luminosity of the source (assumed to be a blackbody), and the inner hydrogen density. The filling factor was set at 0.2 , typical of what we have found in previous photoionization models of classical novae (see Schwarz et al. 2007, and references within). The abundances of elements with observed emission lines (helium, nitrogen, oxygen and neon) were also allowed to vary while all other elements were left at their solar abundances. The model was tuned using the [Ne II] $(12.81 \mu \mathrm{m}) /[\mathrm{Ne}$ III] $(15.56 \mu \mathrm{m})$ ratio which was sensitive to the effective temperature of the photoionizing source while the [O III $](0.4363 \mu \mathrm{m}) /[\mathrm{O}$ III $](0.5007 \mu \mathrm{m})$ ratio constrain the electron density. The results of the best fit model to the 35 observed line ratios are presented in Table 5 and the best fit model parameters are given in Table 6. With seven free parameters, the best fit model had $\chi^{2}=38$, or a reduced $\chi^{2}$ of 1.36 .

The best fit model had a luminosity of $6.3 \times 10^{36} \mathrm{erg} \mathrm{s}^{-1}$ and was extremely cool, $\mathrm{T}_{\text {eff }}$ $=47,000 \mathrm{~K}$. This low model temperature confirms that the ejecta of V1186 Sco was evolving even more slowly than PW Vul. The infrared spectra of PW Vul taken obtained 270 days after outburst had already revealed the emergence of coronal lines of [Si VI] $(1.961 \mu \mathrm{m})$ and [Mg VIII] $(3.026 \mu \mathrm{m})$ (Williams et al. 1996). There are none of these high ionization coronal lines in the NIR spectrum of V1186 Sco more than a year after outburst. The ejected mass of the best V1186 Sco model was $6.5 \times 10^{-5} \mathrm{M}_{\odot}$. This mass is within a factor of 2 of the typical ejected mass of $\sim 10^{-4} \mathrm{M}_{\odot}$ found for most novae (Schwarz et al. 1997, 2001, 2007) using similiar photoionization modeling techniques.

The helium, nitrogen, oxygen, and neon abundances were all enhanced at 1.3, 50, 4.7, and 1.3 times solar, respectively. In addition, the best fit model with sulfur, argon and iron at solar abundances also predicted emission lines that were not observed, notably [S III] $(0.9069 \mu \mathrm{m}),[\mathrm{Ar}$ III] $(9.0 \mu \mathrm{m})$, and Fe II $(0.62 \mu \mathrm{m})$. Reducing these abundances by a factor of 2 removed the sulfur and argon lines and greatly reduced the predicted Fe II and Fe III lines.

While the oxygen and nitrogen abundances are typical of CO type novae (Schwarz et al. 1997, 2001), the derived neon abundance is interesting. The slight enhancement is not as much as would be expected for a ONeMg type nova ( $\mathrm{Ne} \gtrsim 20$, see Schwarz et al. 2007) but more than expected for an outburst occurring on a pure CO white dwarf. In that case the neon abundance should be similar that of the other heavier elements such as sulfur, argon, and iron which reflect the composition of the secondary. To determine if the derived neon 
enhancement is significant we estimated the uncertainty in the model abundances. Ideally, we would use multiwavelength observations taken during a more evolved epoch to see how well the abundance solution fit. Unfortunately, there are no later data available thus we used the method outlined in Schwarz et al. (2001) to estimate the uncertainties in the abundances. The technique assumes that the model parameters were not correlated. Holding all other parameters fixed at their values in the best fit model, one abundance at a time was adjusted until the reduced $\chi^{2}$ of the model increased to 2. This approach provides an approximate $3 \sigma$ uncertainty for each abundance. The neon abundance range from this method is 0.7 1.9 times solar (see Table 6) which is still greater than the upper limit of 0.5 times solar for the sulfur, argon, and iron abundances. Note that the derived neon abundance is relative to the solar neon value of Grevesse \& Noels (1993) which is similar to the new results of Cunha et al. (2006). Both neon abundances are significantly higher, approximately 0.3 dex, than the recent solar neon values of Lodders (2003) and Asplund (2005). If the solar neon abundance is closer to that determined by Lodders (2003) and Asplund (2005) then our derived neon abundance is even more impressive.

The high resolution line profiles from the Keck observations indicate that the H I profiles (all series from $n=2,3$, and 4) have the same asymmetry throughout the observed period. The identical nature of all these transitions implies that the optical depth is likely small enough to insure the correctness of our extinction inferred in Section 3. The H I profile similarities is confirmed by the asymmetries evident in the Spitzer high resolution spectra although the profiles are not well resolved. In contrast, the forbidden lines, for instance [Ne II] $2.089 \mu \mathrm{m}$, display nearly symmetric profiles (Fig. 7). The shell thus appears, as in many novae observed with sufficient spectral resolution, to be quite fragmented with density concentrations isolated in velocity (and therefore in space) within the ejecta. In this case, rather than representing an abundance inhomogeneity or an effect of the optical depth, the profiles can best be explained as local knots of emission at higher than quenching density. While the density variation is likely small (a factor of 20 to $50 \%$ ), this will need to be properly included in any further analysis for the abundance mixing within the ejecta.

Fitting the Cloudy model spectral energy distribution to the dereddened E2 spectra also provides an estimate on the distance. However, before this can be done the observed E2 spectra must be placed on a common flux point since they were obtained at times when the visual light curve wasn't constant. The visual light curve shows that when the Spitzer spectra were obtained, V1186 Sco was about 0.7 magnitude brighter than when the optical spectrum was taken. Therefore the relative fluxes of the Spitzer spectra were reduced by a factor of 1.85. No relative flux adjustments were applied to the NIR spectrum since there are no light curve measurements at that time. Fig. 8 shows the model spectral energy distribution which fits the scaled and dereddened multiwavelength data assuming a distance 
of $5.5 \mathrm{kpc}$. This distance determination is farily robust as the model fits the emission line ratios and the continuum which are both sensitive to the reddening. If the luminosity of the WD was not constant during the E2 epoch but rather oscillating during the early outburst (e.g. the super soft source X-ray luminosities of V4743 Sgr (Ness et al. 2003) and RS Oph (Osborne et al. 2006a, b. c) ) then the derived distance is a lower limit. As a check we apply our Cloudy luminosity to a different epoch, namely visual maximum. With $\mathrm{V}_{\max }=9.6$, an $\mathrm{E}(\mathrm{B}-\mathrm{V})=0.45$, and assuming a bolometric correction of 0 at maximum gives a distance of $5.4 \mathrm{kpc}$, consisitent with our previous value. With the uncertainty in the reddening, which is likely the largest source of uncertainty, we adopt a distance of $5.5 \pm 0.5 \mathrm{kpc}$ and a $\mathrm{M}_{V}$ of $-5.5 \pm 0.5 \mathrm{mag}$ for V1186 Sco.

We also attempted to find better fitting Cloudy models to the E2 spectra with larger $\mathrm{E}(\mathrm{B}-\mathrm{V})$ values as indicated by the Schlegel et al. (1998) reddening map. Increasing E(B-V) to 0.6 the $\chi^{2}$ of the best fit model was much higher, $\sim 75$, and the fit of the model continuum to the data was significantly worse, particularly at shorter wavelengths. Assuming the PW $\mathrm{Vul} \mathrm{M}_{V}$ of -6.7 , the distance at this reddening is $7.7 \mathrm{kpc}$. However, to match the dereddened spectra to the best fit model at $7.7 \mathrm{kpc}$ also requires invoking a covering factor 6 of 0.6 which was not required in the low $\mathrm{E}(\mathrm{B}-\mathrm{V})$ model. Cloudy models fits to spectra dereddened greater than this become progressively worse and indicate that our adopted $\mathrm{E}(\mathrm{B}-\mathrm{V})$ is likely correct.

\section{DISCUSSION}

The oxygen and nitrogen enhancements predicted by the photionization analysis are expected from a thermonuclear runaway in the accretion plus WD material mixture on a CO white dwarf. Carbon may also be enhanced but the best lines to determine this are in the UV. The slight enhancement of neon is probably due to the small amount of ${ }^{22} \mathrm{Ne}$ in the WD which is produced by helium burning while still a giant (Livio \& Truran 1994). Nucleosynthesis models that incorporate some ${ }^{22} \mathrm{Ne}$ in the CO WD composition will produce ejecta with slightly enhanced neon abundances (see Fig. 1 in José \& Hernañz 1998). The presence of the other heavy elements likely reflects that of the secondary star since these elements are only created under the extremely high temperature thermonuclear runaways found on very massive WDs.

V1186 Sco was the first CO-type nova to reveal the strong [Ne II] line common in ONeMg novae using moderate resolution spectroscopic techniques, but other CO novae may have have also shown this line if observed early enough in their evolution prior to the condensation of

\footnotetext{
${ }^{6}$ The fraction ejecta surface area as seen by the photoionizing source.
} 
optically thick dust shells. Evans et al. (1997) reported that [Ne II] line was weak in the day 396 spectrum of V705 Cas and may have been present as early as day 251. The [Ne III] line was also observed in V1425 Aql 650 days after outburst when the nova was still in a high ionization state (Lyke et al. 2003). Spitzer observations of future early CO novae spectra will provide additional estimates of the amount of neon enhancement. These results may even provide observational clues as to the nature of the mixing mechanism and the compositions of CO WDs.

The absolute $\mathrm{V}$ band magnitude determined from the photoionization analysis is very faint at $-5.5 \pm 0.5$. This is consistent with the results of Duerbeck (1981) who found that $<\mathrm{M}_{V}>=-6.35 \pm 0.5$ for historic galactic novae of the B-D light curve class. Our results imply that V1186 Sco is one of the faintest galactic nova ever discovered. In addition, the faint absolute magnitude of V1186 Sco shows that the results of MMRD relationships are suspect for novae with irregular light curves.

\section{CONCLUSION}

V1186 Sco was observed with Spitzer during two epochs during its first year of outburst as part of a Cycle 1 ToO program for classical novae. Its general properties are summarized in Table 7. The Spitzer spectra were combined with ground based optical and NIR spectra obtained during the same epochs. The spectra are typical of a slowly evolving CO-type nova and unambiguously reveal the detection of [Ne II] $(12.81 \mu \mathrm{m})$ in this class of outburst, a highly uncommon event. The reddening toward V1186 Sco, estimated using the intrinsic $(\mathrm{B}-\mathrm{V})$ colors and theoretical hydrogen and $\mathrm{O}$ I line ratios, converged at $\mathrm{E}(\mathrm{B}-\mathrm{V})=$ $0.45 \pm 0.1$. The well sampled $\mathrm{V}$ band light curve displayed a slow decline but with many secondary peaks that call into question many of the properties derived via $t_{2}$ and $t_{3}$ times such as the absolute brightness at maximum. The distance derived from the MMRD relationship put V1186 Sco on the other side of the Galactic center indicating that the MMRD accuracy is suspect for slow novae with irregular light curves.

Photoionization modeling of the data indicates a relatively low mass ejection event consisting of material enhanced in helium, nitrogen, oxygen and some neon. However, the sulfur, argon and iron abundances must be substantially subsolar to explain their lack of prominent lines in the observed spectra. The enhanced oxygen and nitrogen, the erratic light curve, and the low velocity expansion velocities are all consistent with the presence of a CO white dwarf in the system. The amount of neon enhancement $(1.3 \pm 0.6 \mathrm{Ne} / \mathrm{Ne} \odot)$ is not nearly as high as expected from an outburst on a ONeMg WD. Rather the neon is likely intrinsic to the WD and mixed with the accreted material during the thermonuclear 
runaway. The distance derived from the best fit photoionization model provides a more consistent distance of $5.5 \pm 0.5 \mathrm{kpc}$ and a very faint $\mathrm{M}_{V}$ of $-5.5 \pm 0.5$ mags.

We acknowledge with thanks the variable star observations from the AAVSO International Database contributed by observers worldwide and used in this research. This work is based in part on observations made with the Spitzer Space Telescope, which is operated by the Jet Propulsion Laboratory, California Institute of Technology, under NASA contract 1407. The Spitzer Cycle 1 team is supported in part by NASA through contracts 1267992 issued by JPL Caltech. This publication also makes use of data products from the Two Micron All Sky Survey, which is a joint project of the University of Massachusetts and the Infrared Processing and Analysis Center/California Institute of Technology, funded by the National Aeronautics and Space Administration and the National Science Foundation. MFB is grateful to the UK PPARC for the provision of a Senior Fellowship. SGS acknowledges partial support from NASA, NSF, and Spitzer. RDG acknowledges support from Spitzer contracts 1256406 and 1215746 issued by JPL Caltech to the University of Minnesota.

Facilities: Spitzer (IRS), IRTF (SpeX), Keck:II (NIRSPEC), Bok (B\&C spectrograph), MMT (Blue channel spectrograph), Liverpool:2m (RATCam), UKIRT (UIST, CGS4) 


\section{REFERENCES}

Asplund, M. 2005, ARA\&A, 43, 481

Bevington, P. R., \& Robinson, D. K., 1992, Data Reduction and Error Analysis for the Physical Sciences, (New York: McGraw-Hill)

Cunha, K., Hubeny, I., \& Lanz, T. 2006, ApJ, 647, L143

Cushing, M. C., Vacca, W. D., \& Rayner, J. T. 2004, PASP, 116, 362

della Valle, M., \& Livio, M. 1995, ApJ, 452, 704

Downes, R. A., \& Duerbeck, H. W. 2000, AJ, 120, 2007

Duerbeck, H. W. 1981, PASP, 93, 165

Ferland, G.J., Korista, K.T., Verner, D.A., Ferguson, J.W., Kingdon, J.B. \& Verner, E.M. 1998, PASP, 110, 761

Evans, A., Gehrz, R. D., Geballe, T. R., Woodward, C. E., Salama, A., Antolin Sanchez, R., Starrfield, S. G., Krautter, J., Barlow, M., Lyke, J. E., Hayward, T. L., Eyres, S. P. S., Greenhouse, M. A., Hjellming, R. M., Wagner, R. M., \& Pequignot, D. 2003, AJ, 126,1981

Evans, A., Geballe, T. R., Rawlings, J. M. C., Eyres, S. P. S., \& Davies, J. K. 1997, MNRAS, 292,192

Evans, A., Callus, C. M., Whitlock, P. A., \& Laney, D. 1990, MNRAS, 246, 572

Fujii, M. 2004, IAU Circ., 8369, 3

Gehrz, R. D., Roellig, T. L., Werner, M. W., Fazio, G. G., Houck, J. R., Low, F. J., Rieke, G. H., Soifer, B. T., Levine, D. A., \& Romana, E. A. 2007, Rev. Sci. Instr., 78, 011302

Gehrz, R. D., Woodward, C. E., et al. 2007 submitted to ApJ

Gehrz, R. D., Truran, J. W., Williams, R. E., \& Starrfield, S. G. 1998, PASP, 110, 3

Gehrz, R. D., Harrison, T. E., Ney, E. P., Matthews, K., Neugebauer, G., Elias, J., Grasdalen, G. L., \& Hackwell, J. A., 1988, ApJ, 329, 894

Gehrz, R. D., Grasdalen, G. L., \& Hackwell, J. A. 1985, ApJ, 298, L47 
Greenhouse, M. A., Grasdalen, G. L., Hayward, T. L., Gehrz, R. D., \& Jones, T. J. 1988, AJ, 95, 172

Grevesse, N. \& Noels, A., 1993, in Prantzos, de. N., Vangioni-Flam, E., \& Casse, M., eds, Origin \& Evolution of the Elements. Cambridge Univ. Press, Cambridge, p. 15

Hayward, T. L., Gehrz, R. D., Miles, J. W., \& Houck, J. R. 1992, ApJ, 401, L101

Hoffleit, D., \& Jaschek, C. 1982, The Bright Star Catalogue, New Haven: Yale University Observatory (4th edition), 1982

Houck, J. R., et al. 2004, ApJS, 154, 18

Hummer, D. G., \& Storey, P. J. 1987, MNRAS, 224, 801

José, J., \& Hernañz, M. 1998, ApJ, 494, 680

Joyce, R. R. 1992, ASP Conf. Ser. 23: Astronomical CCD Observing and Reduction Techniques, 23,258

Koornneef, J. 1983, A\&A, 128, 84

Kurucz, R. L. 1991, Precision Photometry: Astrophysics of the Galaxy, 27

Kurucz, R. 1994, Solar abundance model atmospheres for 0,1,2,4,8 km/s. Kurucz CD-ROM No. 19. Cambridge, Mass.: Smithsonian Astrophysical Observatory, 1994., 19

Livio, M., \& Truran, J. W. 1994, ApJ, 425, 797

Lodders, K. 2003, ApJ, 591, 1220

Lyke, J. E., et al. 2003, AJ, 126, 993

Lynch, D. K., et al. 2006, ApJ, 638, 987

McLean, I. S., et al. 1998, Proc. SPIE, 3354, 566

Mountain, C. M., Robertson, D. J., Lee, T. J., \& Wade, R. 1990, Proc. SPIE, 1235, 25

Munari, U., et al. 1996, A\&A, 315, 166

Payne-Gaposchkin, C. 1957, in "The Galactic Novae", (New York: Dover)

Ness, J.-U., et al. 2003, ApJ, 594, L127

Osborne, J., et al. 2006, The Astronomer's Telegram, 764, 1 
Osborne, J., et al. 2006, The Astronomer's Telegram, 770, 1

Osborne, J., et al. 2006, The Astronomer's Telegram, 801, 1

Pojmanski, G., 2004, IAU Circ., 8369, 1

Ramsay Howat, S. K., et al. 2004, Proc. SPIE, 5492, 1160

Rayner, J. T., et al. PASP, 115, 362

Rudy, R., Erwin, P., Rossano, G. S., \& Puetter, R. C. 1991, ApJ, 383, 344

Schlegel, D. J., Finkbeiner, D. P., \& Davis, M. 1998, ApJ, 500, 525

Schmidt, G. D., Weymann, R. J., \& Foltz, C. B. 1989, PASP, 101, 713

Schwarz, G. J., Starrfield, S., Shore, S. N., \& Hauschildt, P. H. 1997, MNRAS, 290, 75

Schwarz, G. J., Shore, S. N., Starrfield, S., Hauschildt, P. H., Della Valle, M., \& Baron, E. 2001, MNRAS, 320, 103

Schwarz, G. J. 2002, ApJ, 577, 940

Schwarz, G. J. Shore, S. N., Starrfield, S., Vanlandingham, K. M., 2007, ApJ, 657, 453

Steele, I. A., et al. 2004, Proc. SPIE, 5489, 679

van den Bergh, S., \& Younger, P. F. 1987, A\&AS, 70, 125

Vanlandingham, K. M., Schwarz, G. J., Shore, S. N., Starrfield, S., \& Wagner, R. M. 2005, ApJ, 624, 914

Waagen, E. O. 2005, Observations from the AAVSO International Database, private communication.

Warner, B. 1995, Cataclysmic Variable Stars (Cambridge: Cambridge Univ. Press)

Werner, M. W., et al. 2004, ApJS, 154, 1

Williams, P. M., Longmore, A. J., \& Geballe, T. R. 1996, MNRAS, 279, 804

Woodward, C. E., Gehrz, R. D., Jones, T. J., Lawerence, G. F. \& Skrutskie, M. F. 1997, ApJ, 477, 817

Woodward, C. E., Gehrz, R. D., Jones, T. J., \& Lawerence, G. F. 1992, ApJ, 384, L41 
Table 1. LIVERPOOL ROBOTIC 2-m OPTICAL PHOTOMETRY

\begin{tabular}{ccccc}
\hline \hline Date (UT) & MJD & V & r' & i' \\
\hline 12 Aug 2004 & 53299.8667 & $12.46 \pm 0.03$ & $\ldots$ & $10.73 \pm 0.08$ \\
17 Mar 2005 & 53446.2693 & $13.37 \pm 0.05$ & $12.62 \pm 0.04$ & $13.18 \pm 0.03$ \\
18 Mar 2005 & 53447.2670 & $13.25 \pm 0.08$ & $12.58 \pm 0.04$ & $13.14 \pm 0.05$ \\
26 Mar 2005 & 53455.2630 & $13.43 \pm 0.04$ & $12.59 \pm 0.04$ & $13.14 \pm 0.06$ \\
29 Apr 2005 & 53489.1485 & $\ldots$ & $12.48 \pm 0.12$ & $13.03 \pm 0.10$ \\
30 Apr 2005 & 53490.1502 & $13.47 \pm 0.06$ & $12.71 \pm 0.05$ & $13.17 \pm 0.08$ \\
03 May 2005 & 53493.1424 & $13.45 \pm 0.05$ & $12.74 \pm 0.02$ & $13.23 \pm 0.04$ \\
18 May 2005 & 53508.1003 & $13.41 \pm 0.02$ & $12.94 \pm 0.02$ & $13.34 \pm 0.25$ \\
20 May 2005 & 53510.0965 & $13.46 \pm 0.08$ & $12.91 \pm 0.05$ & $13.48 \pm 0.03$ \\
28 May 2005 & 53518.0159 & $13.53 \pm 0.05$ & $12.80 \pm 0.04$ & $13.37 \pm 0.09$ \\
29 May 2005 & 53519.0148 & $13.43 \pm 0.07$ & $12.83 \pm 0.11$ & $13.40 \pm 0.09$ \\
\hline
\end{tabular}


Table 2. SPECTROSCOPIC OBSERVATION LOG

\begin{tabular}{|c|c|c|c|c|c|}
\hline $\begin{array}{c}\text { Obs. Date } \\
\text { (UT) }\end{array}$ & $\begin{array}{l}\mathrm{Age}^{\mathrm{a}} \\
\text { (d) }\end{array}$ & $\begin{array}{l}\text { MJD } \\
\text { (d) }\end{array}$ & Telescope & Instrument & $\begin{array}{c}\text { Wavelength } \\
\quad(\mu \mathrm{m})\end{array}$ \\
\hline \multicolumn{6}{|c|}{ E1 } \\
\hline 22 Aug 2004 & 50.1 & 53239.765 & Keck II & NIRSPEC (N-1) & $0.93-1.14$ \\
\hline 23 Aug 2004 & 51.1 & 53240.733 & Keck II & $\operatorname{NIRSPEC~}\left(\mathrm{K}_{l}\right)$ & $2.15-2.57$ \\
\hline 23 Aug 2004 & 51.1 & 53240.758 & Keck II & $\operatorname{NIRSPEC~}\left(\mathrm{K}_{s}\right)$ & $1.82-2.24$ \\
\hline 23 Aug 2004 & 51.1 & 53240.767 & Keck II & NIRSPEC $(J)$ & $1.14-1.42$ \\
\hline 27 Aug 2004 & 55.5 & 53244.489 & Spitzer & IRS & $5-35$ \\
\hline 29 Aug 2004 & 57.3 & 53246.331 & Spitzer & IRS & $5-35$ \\
\hline 31 Aug 2004 & 59.3 & 53248.310 & Spitzer & IRS & $5-35$ \\
\hline 02 Sept 2004 & 61.9 & 53250.854 & IRTF & SpeX & $0.85-2.5$ \\
\hline 03 Sept 2004 & 62.1 & 53251.734 & UKIRT & UIST (L) & $2.92-3.64$ \\
\hline 03 Sept 2004 & 62.1 & 53251.756 & UKIRT & UIST (L') & $3.52-4.15$ \\
\hline 03 Sept 2004 & 62.1 & 53251.776 & UKIRT & UIST (HK) & $1.40-2.50$ \\
\hline 04 Sept 2004 & 63.1 & 53252.724 & UKIRT & $\operatorname{UIST}(\mathrm{M})$ & $4.46-5.23$ \\
\hline 04 Sept 2004 & 63.1 & 53252.769 & UKIRT & CGS4 (Z) & $0.80-1.10$ \\
\hline 04 Sept 2004 & 63.1 & 53252.792 & UKIRT & CGS4 (J) & $1.02-1.34$ \\
\hline 23 Sept 2004 & 82.1 & 53271.100 & MMT & Blue Channel Spectrograph & $0.34-0.86$ \\
\hline \multicolumn{6}{|c|}{$\mathrm{E} 2$} \\
\hline 21 Mar 2005 & 261.2 & 53450.221 & Spitzer & IRS & $5-35$ \\
\hline 12 Apr 2005 & 283.5 & 53472.470 & Bok 90" & B\&C Spectrograph & $0.35-0.68$ \\
\hline 16 July 2005 & 378.3 & 53567.266 & IRTF & SpeX & $0.85-2.5$ \\
\hline
\end{tabular}

${ }^{a}$ From discovery date 03 July 2004 UT. 
Table 3. THE NIR/SPITZER LINE FLUXES OF THE MOST PROMINENT FIRST EPOCH (2004)

\begin{tabular}{|c|c|c|}
\hline Ion & $\begin{array}{l}\text { Wavelength } \\
\qquad(\mu \mathrm{m})\end{array}$ & $\begin{array}{c}\text { Flux }^{\mathrm{a}} \\
\left(\operatorname{erg~s}^{-1} \mathrm{~cm}^{-2}\right)\end{array}$ \\
\hline \multicolumn{3}{|c|}{ Near-IR } \\
\hline O I & 0.8448 & $(41.1 \pm 0.2) \times 10^{-12}$ \\
\hline H I (9-3) & 0.9226 & $(7.8 \pm 0.1) \times 10^{-12}$ \\
\hline $\mathrm{N} \mathrm{I}$ & 0.9393 & $(66.7 \pm 0.2) \times 10^{-13}$ \\
\hline H I (8-3) & 0.9545 & $(45.2 \pm 0.3) \times 10^{-13}$ \\
\hline $\operatorname{Pa} \delta$ & 1.005 & $(4.6 \pm 0.1) \times 10^{-12}$ \\
\hline$[\mathrm{N} \mathrm{I}]$ & 1.040 & $(57.8 \pm 0.2) \times 10^{-13}$ \\
\hline He I & 1.083 & $(56.0 \pm 0.3) \times 10^{-13}$ \\
\hline $\operatorname{Pa} \gamma$ & 1.094 & $(76.4 \pm 0.2) \times 10^{-13}$ \\
\hline O I & 1.129 & $(45.8 \pm 0.1) \times 10^{-12}$ \\
\hline He I & 1.254 & $(5.5 \pm 0.3) \times 10^{-13}$ \\
\hline $\mathrm{Pa} \beta$ & 1.282 & $(16.0 \pm 0.1) \times 10^{-12}$ \\
\hline O I & 1.317 & $(16.8 \pm 0.3) \times 10^{-13}$ \\
\hline H I (11-4) & 1.681 & $(10.4 \pm 0.1) \times 10^{-13}$ \\
\hline He I & 1.687 & $(12.2 \pm 0.1) \times 10^{-13}$ \\
\hline H I (10-4) & 1.737 & $(15.9 \pm 0.2) \times 10^{-13}$ \\
\hline H I (9-4) & 1.818 & $(17.5 \pm 0.1) \times 10^{-13}$ \\
\hline $\operatorname{Pa} \alpha$ & 1.876 & $(24.5 \pm 0.1) \times 10^{-12}$ \\
\hline $\operatorname{Br} \delta$ & 1.945 & $(2.8 \pm 0.2) \times 10^{-13}$ \\
\hline He I & 2.059 & $(6.6 \pm 0.3) \times 10^{-13}$ \\
\hline He I & 2.113 & $(1.1 \pm 0.3) \times 10^{-13}$ \\
\hline $\operatorname{Br} \gamma$ & 2.166 & $(26.8 \pm 0.2) \times 10^{-13}$ \\
\hline He I & 3.086 & $(7.9 \pm 0.4) \times 10^{-13}$ \\
\hline He I & 3.331 & $(14.2 \pm 0.4) \times 10^{-13}$ \\
\hline $\operatorname{Pf} \gamma^{b}$ & 3.750 & $(14.0 \pm 0.4) \times 10^{-13}$ \\
\hline $\operatorname{Br} \alpha^{b}$ & 4.052 & $(75.6 \pm 0.1) \times 10^{-13}$ \\
\hline$[\mathrm{N} \mathrm{I}]^{\mathrm{b}}$ & 4.650 & $(12.9 \pm 0.1) \times 10^{-13}$ \\
\hline
\end{tabular}


Table 3-Continued

\begin{tabular}{lcl}
\hline \hline \multicolumn{1}{c}{ Ion } & $\begin{array}{c}\text { Wavelength } \\
(\mu \mathrm{m})\end{array}$ & $\begin{array}{c}\text { Flux }^{\mathrm{a}} \\
\left(\mathrm{erg} \mathrm{s}^{-1} \mathrm{~cm}^{-2}\right)\end{array}$ \\
\hline \multicolumn{3}{c}{ Spitzer } \\
\hline \multicolumn{3}{c}{} \\
Hu $\gamma$ & 5.91 & $(1.5 \pm 1.2) \times 10^{-13}$ \\
H I $(12-7)$ & 6.77 & $(8.3 \pm 6.4) \times 10^{-14}$ \\
Pf $\alpha+\mathrm{Hu} \beta$ & 7.46 & $(6.2 \pm 4.6) \times 10^{-13}$ \\
H I $(10-7)$ & 8.76 & $(6.1 \pm 2.2) \times 10^{-14}$ \\
H I $(12-8)$ & 10.50 & $(1.5 \pm 0.2) \times 10^{-13}$ \\
H I $(9-7)$ & 11.31 & $(4.16 \pm 0.2) \times 10^{-13}$ \\
Hu $\alpha$ & 12.37 & $(1.1 \pm 0.03) \times 10^{-12}$ \\
Ne II $]$ & 12.81 & $(3.6 \pm 1.4) \times 10^{-14}$ \\
H I $(13-9)$ & 14.18 & $(7.4 \pm 1.4) \times 10^{-14}$ \\
H I $(10-8)$ & 16.21 & $(2.1 \pm 0.3) \times 10^{-13}$ \\
H I $(8-7)$ & 19.06 & $(3.7 \pm 0.4) \times 10^{-13}$ \\
H I $(13-10)$ & 22.33 & $(2.3 \pm 0.2) \times 10^{-13}$ \\
H I $(9-8)$ & 27.80 & $(2.7 \pm 0.2) \times 10^{-13}$ \\
\hline
\end{tabular}

${ }^{a}$ Observed integrated line flux. Not corrected for reddening.

${ }^{\mathrm{b}}$ Fluxes are weighted average as line profiles are double peaked. 
Table 4. THE LINE FLUXES OF THE MOST PROMINENT LINES IN THE SECOND EPOCH (2005)

\begin{tabular}{|c|c|c|}
\hline Ion & $\begin{array}{l}\text { Wavelength } \\
\qquad(\mu \mathrm{m})\end{array}$ & 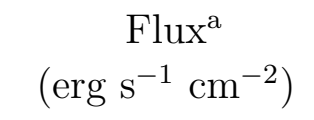 \\
\hline \multicolumn{3}{|c|}{ Optical } \\
\hline $\mathrm{H} \delta$ & 0.4100 & $(2.6 \pm 0.4) \times 10^{-13}$ \\
\hline $\mathrm{H} \gamma$ & 0.4340 & $(4.4 \pm 0.7) \times 10^{-13}$ \\
\hline [O III] & 0.4363 & $(3.5 \pm 0.5) \times 10^{-13}$ \\
\hline He I & 0.4471 & $(8.0 \pm 2.0) \times 10^{-14}$ \\
\hline $\mathrm{H} \beta$ & 0.4868 & $(1.4 \pm 0.1) \times 10^{-12}$ \\
\hline$[\mathrm{O}$ III $]$ & 0.4959 & $(6.5 \pm 0.7) \times 10^{-13}$ \\
\hline [O III $]$ & 0.5007 & $(2.4 \pm 0.2) \times 10^{-12}$ \\
\hline N II & 0.5679 & $(2.4 \pm 0.7) \times 10^{-14}$ \\
\hline$[\mathrm{N}$ II $]$ & 0.5755 & $(2.9 \pm 0.3) \times 10^{-12}$ \\
\hline He I & 0.5876 & $(5.6 \pm 0.8) \times 10^{-13}$ \\
\hline$[\mathrm{O}$ I] & 0.6300 & $(2.5 \pm 0.4) \times 10^{-13}$ \\
\hline He I & 0.6678 & $(1.4 \pm 0.2) \times 10^{-13}$ \\
\hline \multicolumn{3}{|c|}{ Near-IR } \\
\hline O I & 0.8448 & $(4.1 \pm 0.6) \times 10^{-13}$ \\
\hline H I (10-3) & 0.9015 & $(1.5 \pm 0.2) \times 10^{-13}$ \\
\hline H I (9-3) & 0.9229 & $(2.4 \pm 0.4) \times 10^{-13}$ \\
\hline H I (8-3) & 0.9545 & $(4.2 \pm 0.6) \times 10^{-13}$ \\
\hline $\operatorname{Pa} \delta$ & 1.004 & $(4.9 \pm 0.7) \times 10^{-13}$ \\
\hline$[\mathrm{N} \mathrm{I}]$ & 1.040 & $(8.6 \pm 1.7) \times 10^{-14}$ \\
\hline He I & 1.083 & $(1.6 \pm 0.1) \times 10^{-11}$ \\
\hline $\operatorname{Pa} \gamma$ & 1.094 & $(7.6 \pm 0.8) \times 10^{-13}$ \\
\hline $\mathrm{O} \mathrm{I}$ & 1.129 & $(3.0 \pm 0.5) \times 10^{-14}$ \\
\hline He I & 1.197 & $(2.9 \pm 0.5) \times 10^{-14}$ \\
\hline He I & 1.254 & $(5.9 \pm 1.0) \times 10^{-14}$ \\
\hline
\end{tabular}


Table 4-Continued

\begin{tabular}{|c|c|c|}
\hline Ion & $\begin{array}{l}\text { Wavelength } \\
\qquad(\mu \mathrm{m})\end{array}$ & $\begin{array}{c}\text { Flux }^{\mathrm{a}} \\
\left(\mathrm{erg} \mathrm{s}^{-1} \mathrm{~cm}^{-2}\right)\end{array}$ \\
\hline $\mathrm{Pa} \beta$ & 1.282 & $(1.5 \pm 0.2) \times 10^{-12}$ \\
\hline O I & 1.317 & $(4.5 \pm 0.9) \times 10^{-14}$ \\
\hline He I & 1.700 & $(4.7 \pm 1.0) \times 10^{-14}$ \\
\hline $\operatorname{Br} \delta$ & 1.945 & $(1.8 \pm 0.3) \times 10^{-13}$ \\
\hline He I & 2.058 & $(2.1 \pm 0.3) \times 10^{-13}$ \\
\hline He I & 2.113 & $(4.9 \pm 1.0) \times 10^{-14}$ \\
\hline $\operatorname{Br} \gamma$ & 2.166 & $(2.9 \pm 0.4) \times 10^{-13}$ \\
\hline \multicolumn{3}{|c|}{ Spitzer } \\
\hline $\mathrm{Hu} \gamma$ & 5.908 & $(1.5 \pm 1.2) \times 10^{-13}$ \\
\hline H I (12-7) & 6.772 & $(8.3 \pm 6.4) \times 10^{-14}$ \\
\hline $\operatorname{Pf} \alpha+\mathrm{Hu} \beta$ & 7.460 & $(6.2 \pm 4.6) \times 10^{-13}$ \\
\hline H I (10-7) & 8.760 & $(6.1 \pm 2.2) \times 10^{-14}$ \\
\hline H I $(12-8)^{b}$ & 10.500 & $(2.0 \pm 0.6) \times 10^{-14}$ \\
\hline H I $(9-7)^{b}$ & 11.309 & $(8.1 \pm 0.7) \times 10^{-14}$ \\
\hline $\mathrm{Hu} \alpha^{\mathrm{b}}$ & 12.370 & $(2.3 \pm 0.3) \times 10^{-13}$ \\
\hline$[\mathrm{Ne} I \mathrm{II}]^{\mathrm{b}}$ & 12.814 & $(3.5 \pm 0.1) \times 10^{-13}$ \\
\hline H I $(13-9)^{b}$ & 14.183 & $(2.2 \pm 0.6) \times 10^{-14}$ \\
\hline [Ne III] & 15.560 & $(9.9 \pm 0.5) \times 10^{-14}$ \\
\hline H I (10-8) & 16.210 & $(4.9 \pm 1.4) \times 10^{-14}$ \\
\hline H I $(8-7)$ & 19.060 & $(9.3 \pm 1.2) \times 10^{-14}$ \\
\hline H I (13-10) & 22.330 & $(5.3 \pm 0.6) \times 10^{-14}$ \\
\hline H I (9-8) & 27.800 & $(5.2 \pm 0.5) \times 10^{-14}$ \\
\hline
\end{tabular}

${ }^{a}$ Observed integrated line flux. Not corrected for reddening. 
${ }^{\mathrm{b}}$ Weighted average of the SL and SH spectra values. 
Table 5. BEST Cloudy MODEL FITS TO OBSERVED E2 LINE RATIOS

\begin{tabular}{|c|c|c|c|c|}
\hline Line ratio & $\lambda$ & Obs. & Cloudy & $\chi^{2}$ \\
\hline \multicolumn{5}{|c|}{ Optical $^{\mathrm{a}}$} \\
\hline $\mathrm{H} \delta$ & 0.4102 & 0.24 & 0.31 & 1.5 \\
\hline $\mathrm{H} \gamma$ & 0.4340 & 0.38 & 0.52 & 2.2 \\
\hline [O III] & 0.4363 & 0.30 & 0.39 & 1.4 \\
\hline He I & 0.4471 & 0.07 & 0.07 & 0.0 \\
\hline $\mathrm{H} \beta$ & 0.4861 & 1.00 & 1.00 & 0.0 \\
\hline [O III] & 0.4959 & 0.44 & 0.26 & 4.0 \\
\hline [O III $]$ & 0.5007 & 1.62 & 0.79 & 6.5 \\
\hline $\mathrm{N}$ II & 0.5679 & 0.12 & 0.01 & 5.5 \\
\hline$[\mathrm{N}$ II] & 0.5755 & 1.54 & 1.43 & 0.1 \\
\hline He I & 0.5876 & 0.29 & 0.20 & 1.6 \\
\hline$[\mathrm{O} \mathrm{I}]$ & 0.6300 & 0.11 & 0.14 & 0.8 \\
\hline$[\mathrm{O} \mathrm{I}]$ & 0.6363 & 0.05 & 0.04 & 0.1 \\
\hline $\mathrm{H} \alpha+[\mathrm{N} \mathrm{II}]$ & 0.6563 & 4.91 & 4.47 & 0.2 \\
\hline He I & 0.6678 & 0.06 & 0.05 & 0.5 \\
\hline \multicolumn{5}{|c|}{ Near-IR ${ }^{b}$} \\
\hline H I (10-3) & 0.9015 & 0.13 & 0.13 & 0.0 \\
\hline H I (9-3) & 0.9229 & 0.20 & 0.18 & 0.2 \\
\hline H I (8-3) & 0.9546 & 0.35 & 0.23 & 1.8 \\
\hline $\mathrm{Pa} \delta$ & 1.004 & 0.39 & 0.35 & 0.2 \\
\hline$[\mathrm{N} \mathrm{I}]$ & 1.0400 & 0.07 & 0.05 & 0.5 \\
\hline He I & 1.083 & 12.14 & 13.11 & 0.3 \\
\hline $\mathrm{Pa} \gamma$ & 1.094 & 0.57 & 0.55 & 0.0 \\
\hline He I & 1.197 & 0.02 & 0.02 & 0.8 \\
\hline He I & 1.254 & 0.04 & 0.03 & 0.2 \\
\hline
\end{tabular}


Table 5-Continued

\begin{tabular}{|c|c|c|c|c|}
\hline Line ratio & $\lambda$ & Obs. & Cloudy & $\chi^{2}$ \\
\hline $\mathrm{Pa} \beta$ & 1.282 & 1.00 & 1.00 & 0.0 \\
\hline He I & 1.700 & 0.03 & 0.02 & 0.3 \\
\hline $\operatorname{Br} \delta$ & 1.945 & 0.10 & 0.11 & 0.1 \\
\hline He I & 1.954 & 0.01 & 0.02 & 1.7 \\
\hline He I & 2.060 & 0.12 & 0.14 & 0.3 \\
\hline He I & 2.112 & 0.03 & 0.01 & 4.5 \\
\hline $\operatorname{Br} \gamma$ & 2.166 & 0.16 & 0.16 & 0.0 \\
\hline \multicolumn{5}{|c|}{ Spitzer $^{c}$} \\
\hline $\mathrm{Hu} \gamma$ & 5.907 & 0.65 & 0.50 & 1.3 \\
\hline $\mathrm{Pf} \alpha+\mathrm{Hu} \beta$ & 7.458 & 2.70 & 2.75 & 0.0 \\
\hline H I (9-7) & 11.310 & 0.35 & 0.30 & 0.5 \\
\hline Hu $\alpha$ & 12.370 & 1.00 & 1.00 & 0.0 \\
\hline$[\mathrm{Ne} I \mathrm{II}]$ & 12.810 & 1.50 & 1.60 & 0.2 \\
\hline [Ne III] & 15.550 & 0.43 & 0.40 & 0.2 \\
\hline H I (8-7) & 19.060 & 0.40 & 0.40 & 0.0 \\
\hline H I (9-8) & 27.800 & 0.22 & 0.20 & 0.2 \\
\hline
\end{tabular}

${ }^{\text {a Relative to } \mathrm{H} \beta}$

${ }^{b}$ Relative to $\mathrm{Pa} \beta$

${ }^{c}$ Relative to $\mathrm{Hu} \alpha$ 
Table 6. BEST Cloudy MODEL PARAMETERS

\begin{tabular}{|c|c|}
\hline Parameter & Value \\
\hline $\mathrm{T}_{B B}$ & $47,000 \mathrm{~K}$ \\
\hline Source luminosity & $6.3 \times 10^{36} \mathrm{erg} \mathrm{s}^{-1}$ \\
\hline Hydrogen density & $3.2 \times 10^{7} \mathrm{~cm}^{-3}$ \\
\hline Inner radius ${ }^{\mathrm{a}, b}$ & $1.1 \times 10^{15} \mathrm{~cm}$ \\
\hline Outer radius ${ }^{\mathrm{a}, b}$ & $2.2 \times 10^{15} \mathrm{~cm}$ \\
\hline Filling factor ${ }^{\mathrm{b}}$ & 0.2 \\
\hline $\mathrm{He} / \mathrm{He}_{\odot}{ }^{\mathrm{c}}$ & $1.1 \pm 0.3(10)$ \\
\hline $\mathrm{N} / \mathrm{N}_{\odot}^{\mathrm{c}}$ & $49_{-23}^{+34}(5)$ \\
\hline $\mathrm{O} / \mathrm{O}_{\odot}^{\mathrm{c}}$ & $5.1_{-2.8}^{+0.4}(5)$ \\
\hline $\mathrm{Ne} / \mathrm{Ne}_{\odot}{ }^{\mathrm{c}}$ & $1.3 \pm 0.6(2)$ \\
\hline Ejected Mass & $6.5 \times 10^{-5} \mathrm{M}_{\odot}$ \\
\hline Degrees of Freedom & 28 \\
\hline Total $\chi^{2}$ & 38.0 \\
\hline Reduced $\chi^{2}$ & 1.36 \\
\hline
\end{tabular}

Note. - Model had a hydrogen power law of $-3, \mathrm{n}(\mathrm{H}) \propto \mathrm{r}^{-3}$.

${ }^{a}$ Calculated assuming an inner and outer expansion velocity of 500 and 1000 $\mathrm{km} \mathrm{s}^{-1}$, respectively, over 260 days.

${ }^{\mathrm{b}}$ Not a free parameter in the model.

${ }^{c}$ Where Log(Solar number abundances relative to hydrogen) He:-1.0 C: -3.61 $\mathrm{N}:-4.22$ O: -3.34 Ne: $\quad-3.93 \mathrm{Mg}: \quad-$ $4.47 \mathrm{Al}:-5.63 \mathrm{Si}:-4.49 \mathrm{~S}:-4.86 \mathrm{Fe}$ 4.55 (where all abundances taken from Asplund (2005) except for Ne which is 
from Grevesse \& Noels (1993)). All elements not in the table were set to solar values. The number in the parentheses indicates the number of Cloudy lines used in the analysis. 
Table 7. V1186 Sco PROPERTIES

\begin{tabular}{ll}
\hline \hline & \\
\hline Equatorial Coordinates & $\mathrm{RA}=17: 12: 51.3 \mathrm{Dec}=-30: 56: 39(\mathrm{~J} 2000)$ \\
Galactic Coordinates & $l=354.4 \quad b=4.8(\mathrm{G} 2000)$ \\
Date Discovered & 3.146 July $2004(\mathrm{MJD}=53189.146)$ \\
Visual Maximum & 9 July 2004 \\
$\mathrm{~V}_{\text {max }}$ & 9.6 \\
$\mathrm{t}_{2}$ and $\mathrm{t}_{3}$ & 20 and 70 days \\
Line widths & $\sim 500-1000 \mathrm{~km} \mathrm{~s}^{-1}$ \\
$\mathrm{E}(\mathrm{B}-\mathrm{V})$ & $0.45 \pm 0.10$ \\
$\mathrm{M}_{V}^{\text {max }}$ & $-5.5 \pm 0.5$ \\
Distance & $5.5 \pm 0.5 \mathrm{kpc}$ \\
Dust formation? & None detected \\
\hline
\end{tabular}





Fig. 1. - The light curve of V1186 Sco. The V band photometry was taken from VSNET (squares), IAU Circulars (asterisks), AAVSO (diamonds), and our own Liverpool 2-m data (triangles). The left plot shows the early evolution and the right figure shows the light curve over the first year. The lower portion of the left figure shows the $(B-V)$ color evolution from the available data. 


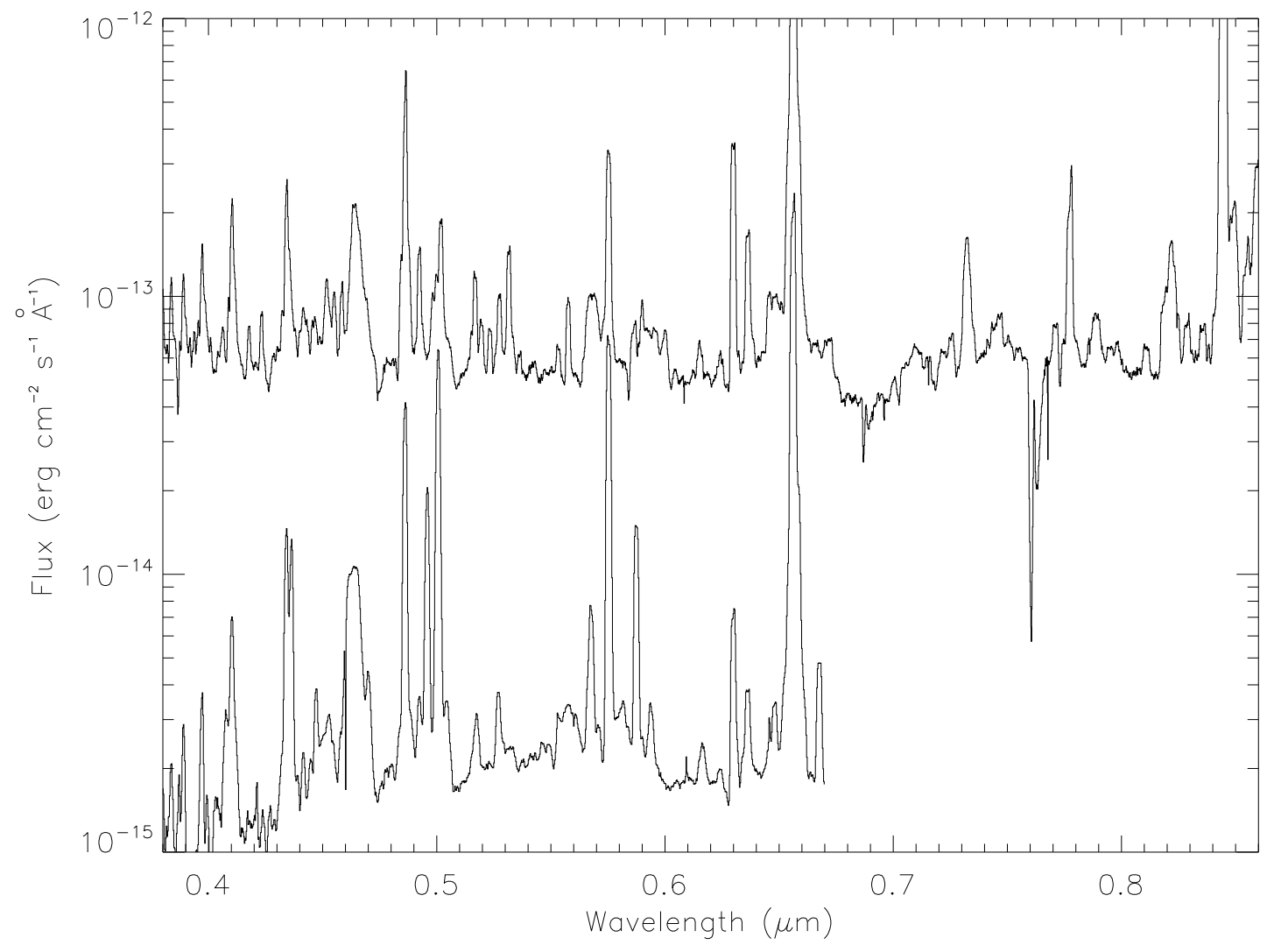

Fig. 2.- The evolution of the optical spectrum during the two observational epochs. The first spectrum ( top line) is typical of a Fe II type novae during the early outburst with narrow, low ionization emission and numerous P Cygni profiles. The second spectrum (bottom line) shows an emerging nebular spectrum. 


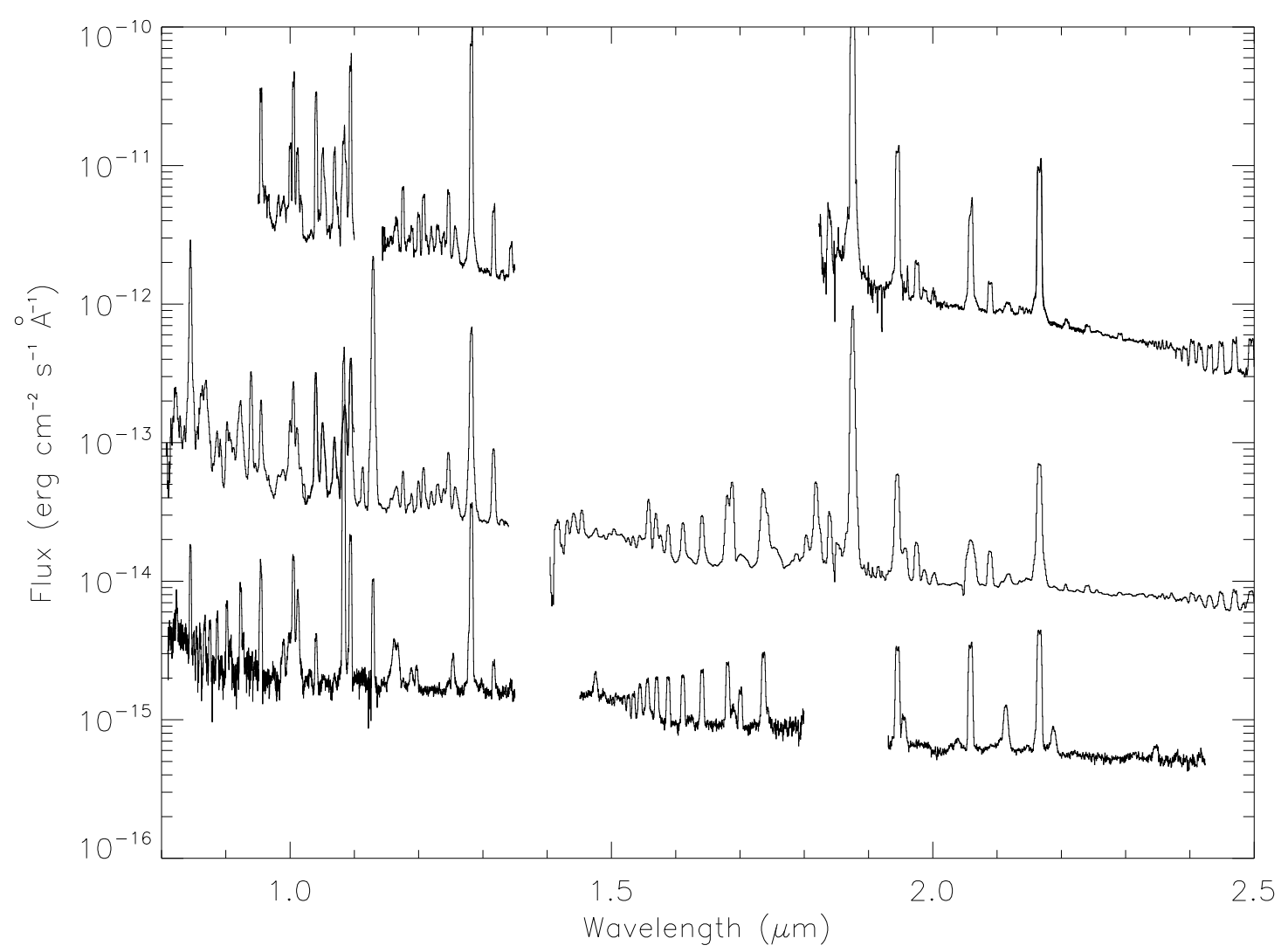

Fig. 3.- The evolution of the near-IR spectrum. The Keck (top line) and UKIRT data (middle line) were obtained early in the outburst in the first epoch. The last SpeX spectrum (bottom line) was taken over a year after outburst. The strongest emission is from He I and O I. The first SpeX spectrum is not displayed because it is of similar signal-to-noise as the middle UKIRT data obtained 1-2 nights later, and exhibiting the same suite of emission lines in the JHK-bands. 


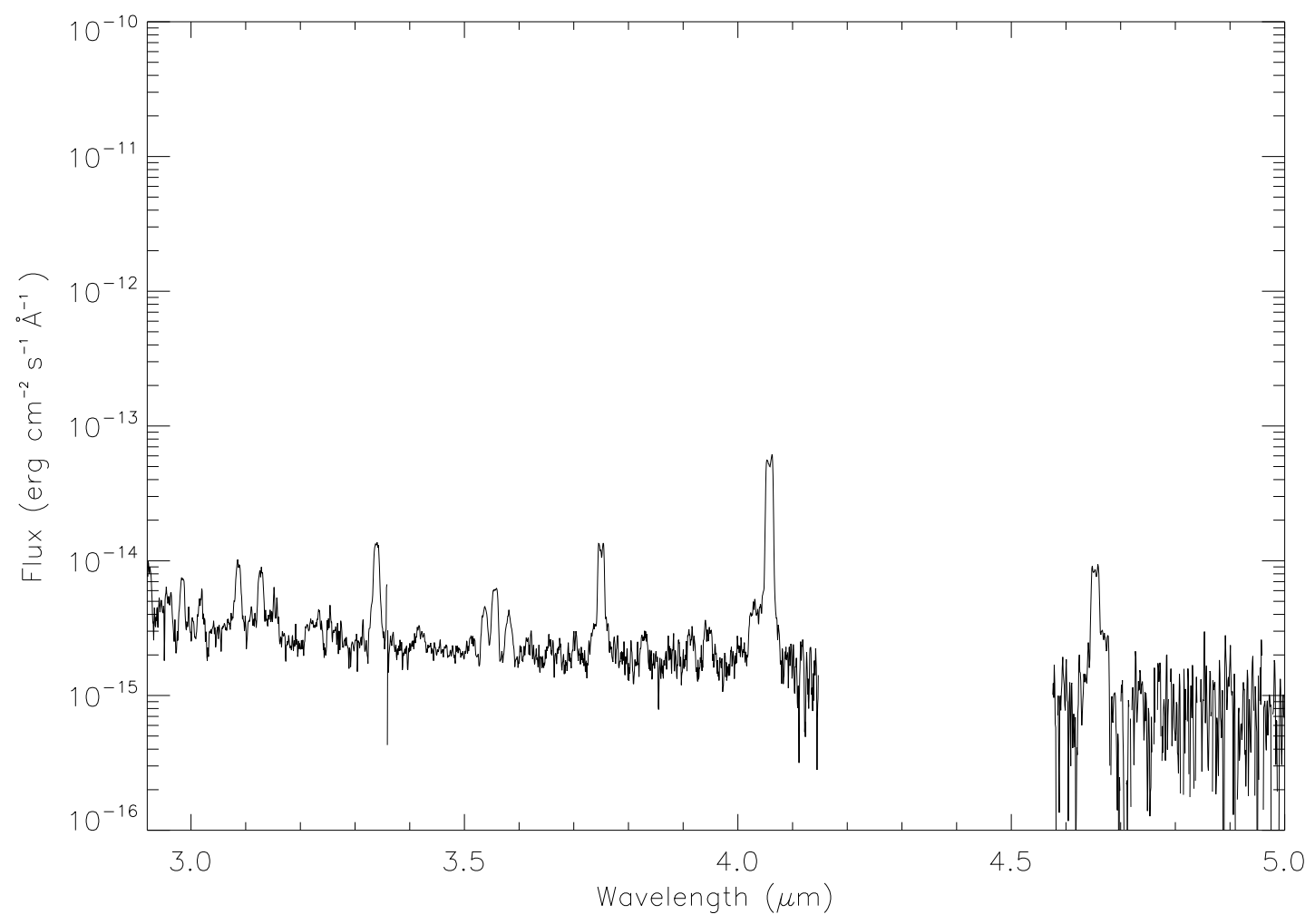

Fig. 4.- The $L M$-band spectra obtained at UKIRT. The spectrum at this epoch (03 September 2004) is dominated by hydrogen recombination lines, [N I], little continuum, and no $3 \mu \mathrm{m}$ dust emission features. The $M$-band spectra is truncated near $5.0 \mu \mathrm{m}$ due to poor signal-tonoise. 

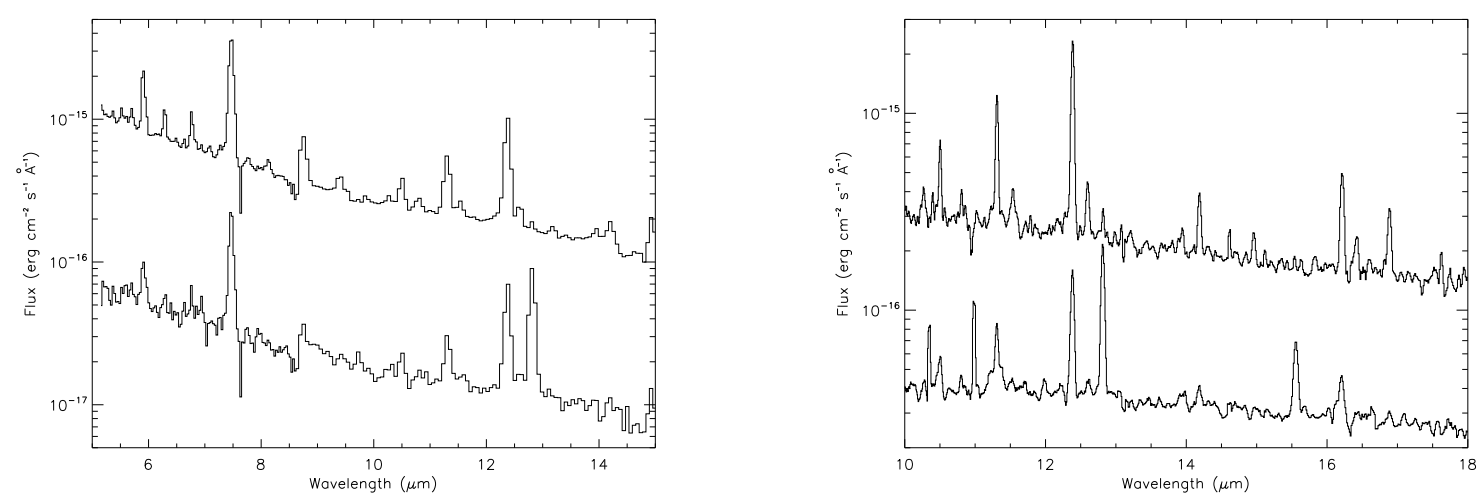

Fig. 5.- The evolution of the Spitzer SL (left panel) and SH (right panel) spectra from the two observational epochs. The first epoch (top line) is dominated by a strong hydrogen recombination spectrum. Weak [Ne II] $(12.81 \mu \mathrm{m})$ can be seen in the SH spectrum. The hydrogen lines are still present at the second epoch (bottom line) but the IR fine-structure lines $([\mathrm{Ne} \mathrm{II}]$ and $[\mathrm{Ne} \mathrm{III}]$ at $15.56 \mu \mathrm{m})$ are now the strongest lines present. The later spectra are offset by 0.3 Jy for clarity.
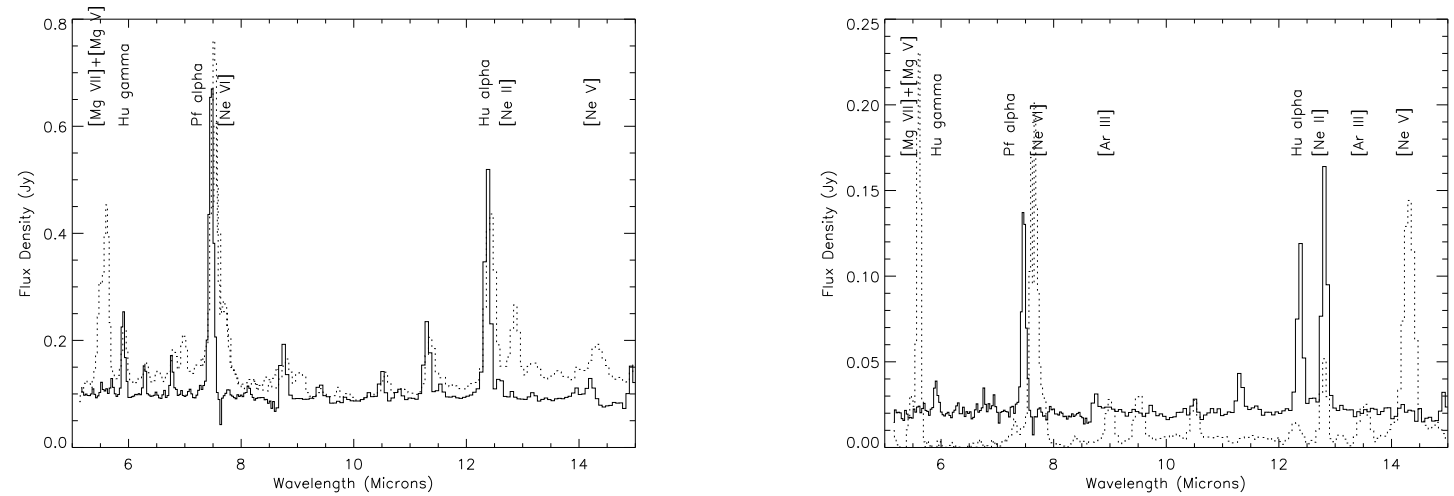

Fig. 6.- Comparison of Spitzer IRS SL line emission evolution in nova V1186 Sco (solid line) and the much faster, ONeMg nova V1187 Sco (dashed line). The left panel compares the 29 August 2004 spectrum of V1186 Sco (E1), with the 28 September 2004 UT spectrum of V1187 Sco observations, while the right panel compares the 21 March 2005 UT (E2) of both novae obtained on the same day. V1186 Sco, in contrast to V1187 Sco, did not develop strong emission lines from metals, such as $\mathrm{Mg}$ or $\mathrm{Ar}$, that are commonly detected in $\mathrm{ONeMg}$ novae. 

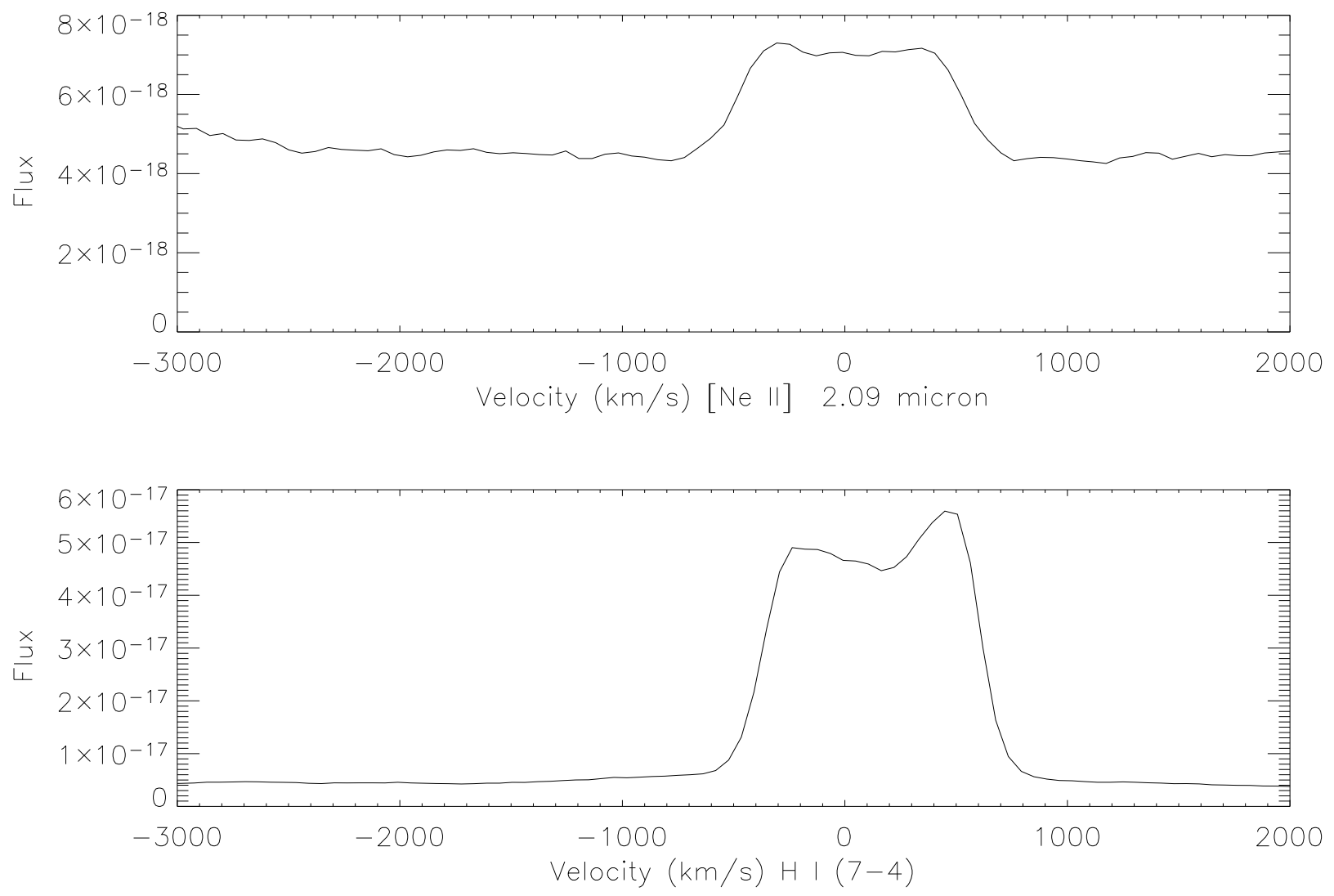

Fig. 7.- Early line profiles of [Ne II] $(2.09 \mu \mathrm{m})$ and H I (7-4) showing the asymmetry between the forbidden lines and the hydrogen lines. 


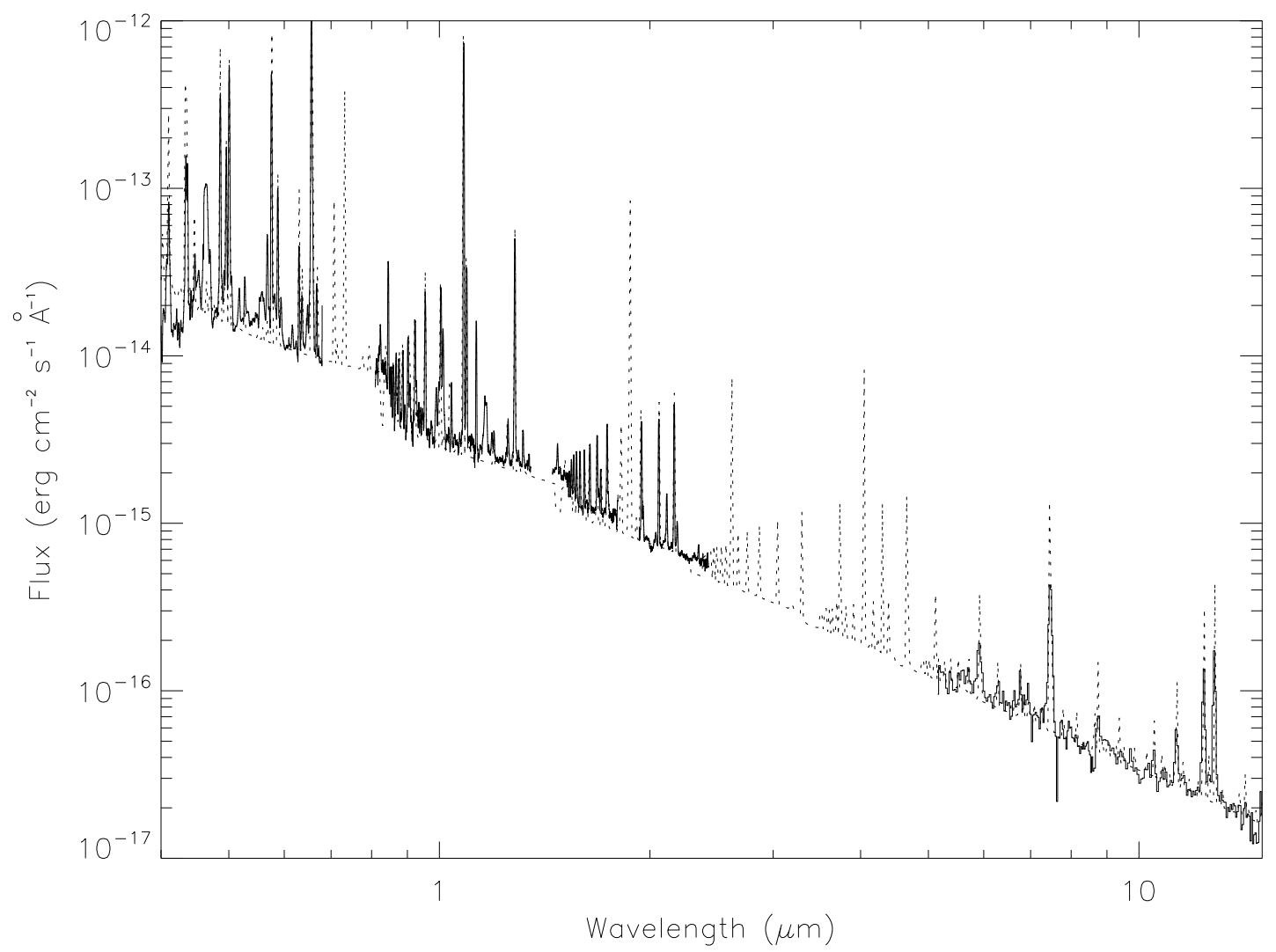

Fig. 8.- Best Cloudy model (dotted line) fit to the optical, NIR, and Spitzer spectra (solid lines) obtained during the E2 epoch. The Spitzer spectra have been scaled to reflect the $\sim 0.7$ mag difference in the visual light curve between the Spitzer and optical observations. The Cloudy model spectral energy distribution fits the observations best at a distance of 5.5 kpc. 